\title{
IMAGE PROCESSING COLOR MODEL TECHNIQUES AND SENSOR NETWORKING IN IDENTIFYING FIRE FROM VIDEO SENSOR NODE
}

\section{S. R. VIJAYALAKSHMI \& S. MURUGANAND}

Department of Electronics and Instrumentation, Bharathiar University, Coimbatore, Tamil Nadu, India

An early warning is an extremely important to reduce loss of life and property from fire. The region of interest is captured using CCD camera and identified by smoke sensor in the wireless sensor node. The color information of interesting region can be obtained with an application of the digital image processing color model algorithms. The fire source is identified according to the acquired characteristics and smoke level. The system is based on the continuous image sampling. The experimental results show that the system can accurately identify and confirm the fire. The video sensor node is designed with the sensors such as MQ2 sensor for smoke sensing, SHT75 sensor for temperature and humidity sensing, OPT101 sensor for light sensing and CCD camera. Alarm is activated only for fire image and fire incidents. By combining sensor output with image output, the false alarm rate is zero and improves the stability. Light detection and analysis is the basis for the fire detection system in this image processing work. In this fire image work color models such as RGB, YMK and HSI are used to separate orange, yellow, and high brightness light from background within given conditions to detect fire. Frame difference is used to analyze and calculate the growth and spread of fire. The accuracy of the system is checked and compared with one another. The amount of data processing can be reduced because of the use of proposed algorithm and thus shorten the execution time and storage.

KEYWORDS: Fire Detection, Fire Characteristics, Smoke Sensor, Video Sensor Node, Color Segmentation, Digital Image Processing, Color Model, RGB, YMK \& HSI
\end{abstract}

Received: Jun 13, 2017; Accepted: Jun 28, 2017; Published: Jul 20, 2017; Paper Id.: IJCSEITRAUG20176

\section{INTRODUCTION}

Even in this modern world, it is very difficult to detect fire in real time. In buildings, fire can spread quickly and cause great loss of life and property. Hence, early warning is an extremely important to reduce it. Smoke detectors and temperature detectors are used widely to protect property and give warning of fire in sensor networking. Using this smoke detection system alone is slow in processing. To cover entire area, many smoke and temperature fire detectors are required. But, it can be helpful to improve the system when combined with the light detection camera image processing technique. In order to find fire at early state and to monitor its growth and spread, the light detection method is helpful, when it is combined with smoke and temperature detection method. This system gets video input from any video camera like web camera. It triggers audible alarm and provides visual images of fire area from video sequences.

Fire has sudden stochastic characteristics. Hence automatic fire monitoring, alarm and control functions are required in modern fire prevention facilities to accomplish real-time monitoring. In recent years, the remote monitoring system based on network video camera provides reliable. The system is comprised of monitoring node, managing command center, wireless transmission, camera and lens, smoke sensor, console control unit and power 
supply system. The fire identification with the digital image processing along with smoke sensing of wireless sensor node can greatly improve the technological content and the automation level of the system, and reduce the labour intensity of personnel.

This paper is organized into five sections: Section I introduce the research project work; Section II describes related works; Section III presents the proposed system in detail; Section IV describes experimental results. Section V about the Conclusion.

\section{RELATED WORK}

S.R. Vijayalakshmi and S.Muruganand [1] discussed about the real time monitoring of wireless fire detection node. M. Morin at.el [2] analyzed about the computer-supported visualization of rescue operations. Xue-gui Wan at.el [3] analyzed about the novel conceptual fire hazard ranking distribution system based on multisensory Technology. Konstantinous at.el [4] dealt about an automated fire detection and alerting application based on satellite and wireless communications. Fatih Erden at.el [5] discussed about the wavelet based flickering flame detector using differential PIR sensors. K.Jayara at.el [6] analysed the adaptive edge detection algorithm for flame and fire images. Shaik Rasool and P.Pandarinath [7] discussed about autoadaptive edge detection algorithm for flame and fire image processing.

\section{IMAGE PROCESSING AND SENSOR NETWORKING FOR FIRE DETECTION}

The system is analyzed from the following four aspects and then make final judgment according to the results of analysis along with smoke sensor node. 1) Color: Fire color (red) is the basic identification basis. 2) Color distribution: From flame cone to outer flame, the color tends to move from white to red. 3) Shape: Fire burning can produce sharp corner, which is called flame current on the edge of the image. Because of the unchanging characteristic, the flame current shape can be considered as an important characteristic to identify fire. 4) The dynamic characteristics: In the process of fire burning, flame's position and area are constantly changing, unlike some fixed shiner like the sun, lamps and any other. Therefore, according to the changes of image pixel values, fire can be distinguished from some other luminous objects. The algorithm is analyzed as image segmentation, noise processing and eliminating, edge detection, peak flame identification, and color model techniques.

\subsection{Image Segmentation}

Image segmentation plays a very important role in image processing. The image segmentation results affect the image of follow-up processing directly. It divides image space into some significant areas according to some characteristics such as gray, spectrum and texture. Classical image segmentation algorithms such as iteration, threshold, segmentation, maximum between cluster variance, the maximum entropy threshold method are entirely depending on gray image which result in the loss of color characteristic of images. Due to this weakness, a new segmentation algorithm based on color characteristics of the image is presented and proposed along with smoke detecting sensor. It can divide and get the flame target better. Most colorful models used today are hardware and application-oriented. In digital image processing, the most common model belongs to hardware-oriented is RGB (red, green, and blue) model which is mainly used in color monitor and a large class of color video camera. HSI (Hue/tonal, Saturation and Intensity/brightness) model is more suitable and explain the color. It also can divide the image information into color and gray to make it easier to apply gray processing technology. The algorithm presented here will use both RGB and HSI models. Firstly, HSI format should be converted into RGB format because the images are all BMP images. Each component has a conversion formula as follows in equation 1: 


$$
I I-\cos ^{-1}\left\{\frac{(2 R-G-B)}{2 \sqrt{((R-G)(R-G)+(R-B)(G-B))}}\right\} ;=\frac{(R+G+B)}{3} ; S=1-3 \times \frac{\operatorname{Min}(R, G, B)}{(R+G+B)}
$$

After getting the HSI values of the image pixel B > G if $\mathrm{H}$ unchanged, B $<\mathrm{G}$ if $\mathrm{H}=360-\mathrm{H}$, and $\mathrm{B}=\mathrm{G}$ if $\mathrm{H}=360$ are judged. The $\mathrm{H}$ values of the flame are within a specific range, only by which the flame target cannot be extracted from the source image accurately. Hence the RGB image segmentation model should be combined with the image segment. To determine whether the color images are in red, the following steps are followed in the algorithm. Its steps are based on 1) Get the three RGB color components; 2) To determine whether the largest component of that is the red component, that is, $\mathrm{R}>\mathrm{G}, \mathrm{R}>\mathrm{B}$; 3) To determine whether the value of the red component has reached a certain amount, that is, $\mathrm{R}>\mathrm{r}(\mathrm{r}$ is the set threshold); 4) Whether the red points are obviously much larger than the second largest value in comparison (That is, $\mathrm{R}>\mathrm{G}, \mathrm{R}>>\mathrm{B})$.

\subsection{Noise Processing and Eliminating}

The image quality decreases with the loss of information in the man-made process. The noise in the image acquisition process affects identifying the characteristics of fire at later stages. Therefore, different kind of noises such as salt pepper is introduced. After that the degraded images need to be smoothed with the median filter method. It is a nonlinear approach used to remove noise. It retains good image details. The median filter is based on replacing the value in digital images with the median value of each point value in neighbor of the point. This filter introduces good distribution of the images on the elimination of all kinds of random noises and also fuzzy edges. Small white areas found in the observed image. This is because of scattering of the beam caused by smoke. To eliminate these noises, black and white noise cancellation and noise reduction are used.

\subsection{Edge Detection}

Extraction of edge information is important in digital image processing for suppressing noise. This paper uses sobel operator to determine the location of the edge. The expression for the sobel operator is given in equation 2 as,

$$
M x=\left[\begin{array}{lll}
-1 & 0 & 1 \\
-2 & 0 & 2 \\
-1 & 0 & 1
\end{array}\right] ; M y=\left[\begin{array}{ccc}
-1 & -2 & -1 \\
0 & 0 & 0 \\
1 & 2 & 1
\end{array}\right]
$$

The edge of the flame will be made into chain code first. To normalize the chain code, circulary shift the chain code to get the smallest and select the first order differential. When the chain code in a certain step takes on an effective rise and fall, a cusp can be obtained. Flame region closed contour collection can be expressed in equation 3 as follows:

$$
C\left(C_{l n}^{m}\right)=\left\{p_{0}, \cdots, p_{k}, p_{0}\right\}
$$

$C_{\ln }^{m}$ is the studied region of the suspicious pixels set; $p_{k}$ is the region on the edge pixels, if the $p_{k}$ point on the curve there is a sharp point, then the following relations equation 4 :

$$
\theta\left(p_{k-1} p_{k}, p_{k+1} p_{k}\right) \leq \theta_{0}
$$

$\theta$ is the angle between two vector functions; $\theta_{0}$ is the angle value which is set.

For flame center to outer, its color mobiles from white to yellow to red generally. The sun, light source of 
interference does not change its color. The pixel value does not change significantly for the sun in the image. According to the physical characteristics of fire, recognition algorithm is proposed as follows. First, check all the suspected objects flame pixel values. Then calculate each pixel value of the proportion of red as in the formula 5:

$$
\text { redratio }(x, y)=\sum_{x y \in m} \frac{R\left(p_{i}(x, y)\right)}{R\left(p_{i}(x, y)\right)+G\left(p_{i}(x, y)\right)+B\left(p_{i}(x, y)\right)}
$$

$R\left(p_{l}(x, y)\right), G\left(p_{i}(x, y)\right), B\left(p_{l}(x, y)\right)$ is the image of three-component red, green and blue and then with that calculating the red pixel value of the variance of the proportion. When the suspicious target is flame means the red side of the margin has larger proportion. When the suspected target of the interference sources such as the sun, lighting, etc., the color did not change significantly and pixel values remained near the average in a volatile. So the red pixel value of the variance of the proportion is small and close to zero. The steps of algorithm implementation are as follows in equation 6 :

Read the suspected flame part of the image pixel value $f(x, y)$, then storage them into the array;

Pixel array for the mathematical expectation $r=E(f(x, y))$;

Pixel array for the variance $c=E(f(x, y)-r)^{2}$; ------(equation 6)

\subsection{Peak Flame Identification}

Flame is the main body of fire. In the collection of flame images the fire is a bright area. So, it is necessary to figure out the flames from the light and understand the irregular changing in the bright areas. The shape of the flame is irregular and changes arbitrarily. But there is a feature of the flame in that some bright areas overlapping but cannot be completely overlapping in two of the suspicious image acquired. When a suspicious light is found named $\mathrm{X}$ collect images and use the above rules to determine the rules of suspicious $\mathrm{Y}$ in bright areas. Principle: If $\mathrm{X}$, Y overlapp windows 0.1 or 0.8 , then it is not the flame. Overlap rate is the overlap part between window $\mathrm{Y}$ and window $\mathrm{X}$ with a value of 1 in accounting for overlap the rate of 1 point. Overlap rate 0.1 is mainly for mobile sources and interference with the light sources. Overlap 0.8 is mainly directed against monitoring of lighting equipments in the region. In the monitoring region using of lighting equipments, the bright window both appear in two adopted images, but when comparing the two, they do not entirely overlap. Thus, the two overlap rate $80 \%$ and it is not a fire which is ruled out as only a possibility of interference of fixed lighting equipment. The sun can be considered as the second situation. Along with the CCD camera, smoke sensor also interfaced in the video mode. The smoke level is compared in some cases to detect fire. If the smoke level exceeds the threshold level, image processing steps are carried out to determine the occurrence of fire.

\subsection{Color Model Techniques}

The proposed fire detection system uses input video from any web camera at real time. The expected fire and background are separated by the HSV/RGB/CMY color segmentation and received fire location frames. The consecutive fire location frames are compared to check fire growth and spread. If its growth continues over frame by frame the alarm is triggered and computer system displays the fire growth location. Color segmentation is used to segment fire from its background. Fire color consists of orange, yellow and brightness. HSV/RGB/YMC color models are used to separate flame from the background. It is used to detect information related with color and brightness. The condition to detect fire is given in equation 7 . 


$$
f(x, y)=\left\{\begin{array}{c}
1, \text { if }((0.02<H(x, y)<0.30) \text { and } \\
(0.20<S(x, y)<1.00) \text { and } \\
(0.98<V(x, y)<1.00)) \\
\text { if }(R(x, y) \gg G(x, y)>B(x, y)) \\
\text { or }(Y(x, y) \rightarrow M(x, y)>C(x, y) \\
0, \text { otherwise }
\end{array}\right.
$$

Where $\mathrm{H}(\mathrm{x}, \mathrm{y}), \mathrm{S}(\mathrm{x}, \mathrm{y})$, and $\mathrm{V}(\mathrm{x}, \mathrm{y})$ are hue, saturation, and intensity value at the spatial location (x, y) respectively in the HSV or HSI color model. $\mathrm{R}(\mathrm{x}, \mathrm{y}), \mathrm{G}(\mathrm{x}, \mathrm{y})$ and $\mathrm{B}(\mathrm{x}, \mathrm{y})$ are red, green and blue at the spatial location $(\mathrm{x}, \mathrm{y})$ respectively in the $\mathrm{RGB}$ color model. $\mathrm{Y}(\mathrm{x}, \mathrm{y}), \mathrm{M}(\mathrm{x}, \mathrm{y})$ and $\mathrm{C}(\mathrm{x}, \mathrm{y})$ are yellow, magenta and cyan at the spatial location $(\mathrm{x}, \mathrm{y})$ respectively in the CMY color model. Each frame of the video input is processed to follow the above mentioned conditions and generate output fire location images. Each pixel of the fire location images contains either 1 or 0 . The pixel value 1 means the flame pixel. The pixel value 0 means the non-flame pixel.
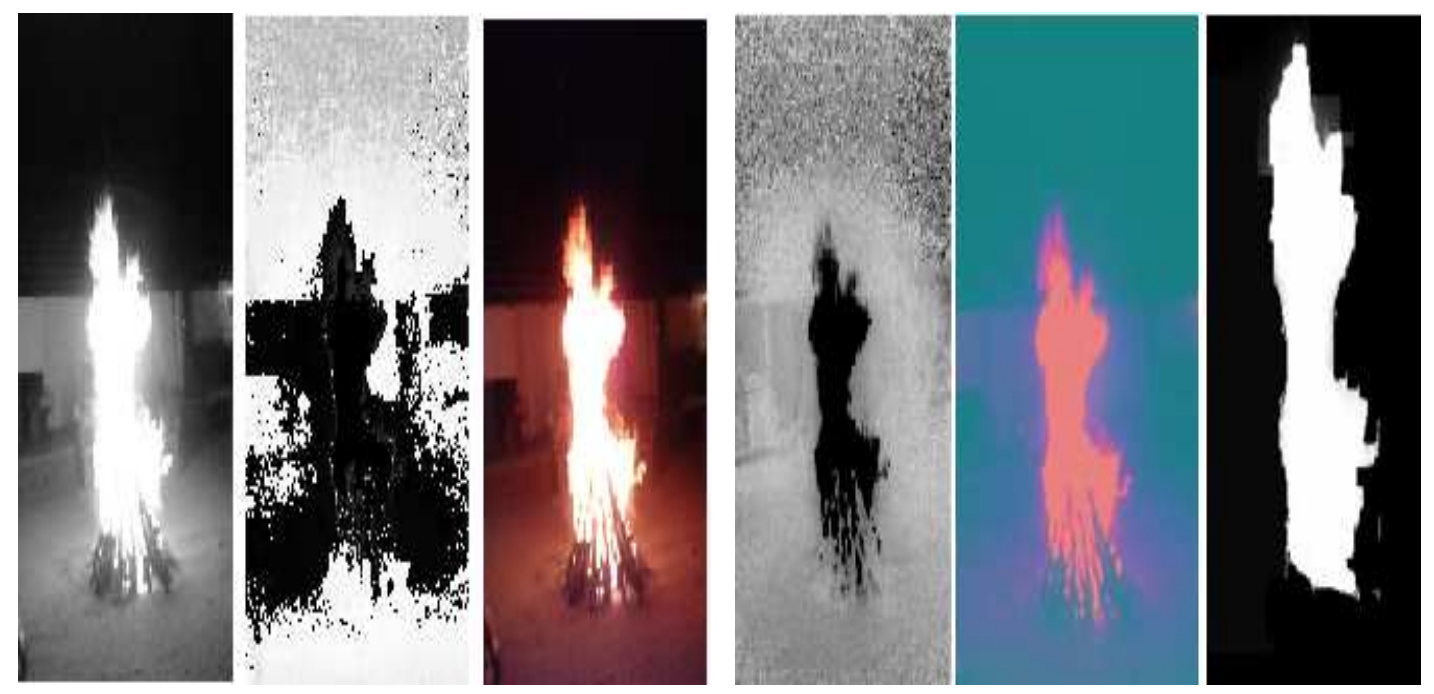

(A) V O/P Image (B) H O/P Image (C) RGB O/P Image (D) S O/P Image

(E) Ycbcr O/P Image (F) Fire Location O/P Image

\section{Figure 1: Color Segmentation Technique}

Figure 1 shows images from the developed color segmentation technique. The figure (f) is the corresponding fire location image output with two colors, white and black. White color represents the pixel value 1 and black color represents the pixel value 0 and the white area represents the fire location.

The developed color segmentation technique can effectively detect fire flame in real-time fire video sequences. The accuracy of this technique is more than $80 \%$. However, it is possible for fault detection if there are lighted candles, lighted matches, orange clothes, or other objects with bright orange color in the video sequences. Therefore, the technique has been further improved by checking fire growth and spread of fire and combining the process with the smoke detection. A noise reduction algorithm is applied to reduce the possible noise that can cause false detection. After that fire growth is checked by the algorithm as shown in figure 4 .

The two consecutive fire location images are selected. White pixels in each image are counted. Then, the difference between the numbers of white pixels in each image is calculated by the numbers of white pixels in the next image subtracted by the numbers of white pixels in the base image. In order to check fire growth, the data difference 
between the two frames should be positive and large enough to be able to detect fire growth. Therefore, it is selected $13 \%$ of data difference and based images. If these conditions are correct, the system activates the alarm sound and provides image notification at fire flame location in the video sequences.

\section{RESULTS AND ANALYSIS}

Through the result of the system testing, the fire identified automatically. The system have high sensitivity and low false alarm rate. It also have a very good ability of learning and adapting itself. It can recognize the complex situation of the non-fire and the anti-interference strongly. The system also has advantages such as low cost, wide monitoring range, quick response time and no need to change the network bus. The system reduces the false alarm rate and improves the stability by using the temperature and smoke sensors in the node. Alarm is activated only for fire image and fire incidents. The video sensor node is as shown in figure 2. The video sensor node is designed with the sensors such as MQ2 sensor for smoke sensing, SHT75 sensor for temperature and humidity sensing, OPT101 sensor for light sensing and CCD camera. All the sensors are interfaced with the microcontroller with minimum interfacing components. XBeePro transceiver IC is interfaced with the microcontroller for transmitting and receiving information to and from the control station. The table 1 shows the smoke sensor output combined with image to detect fire. So the false alarm rate is zero.

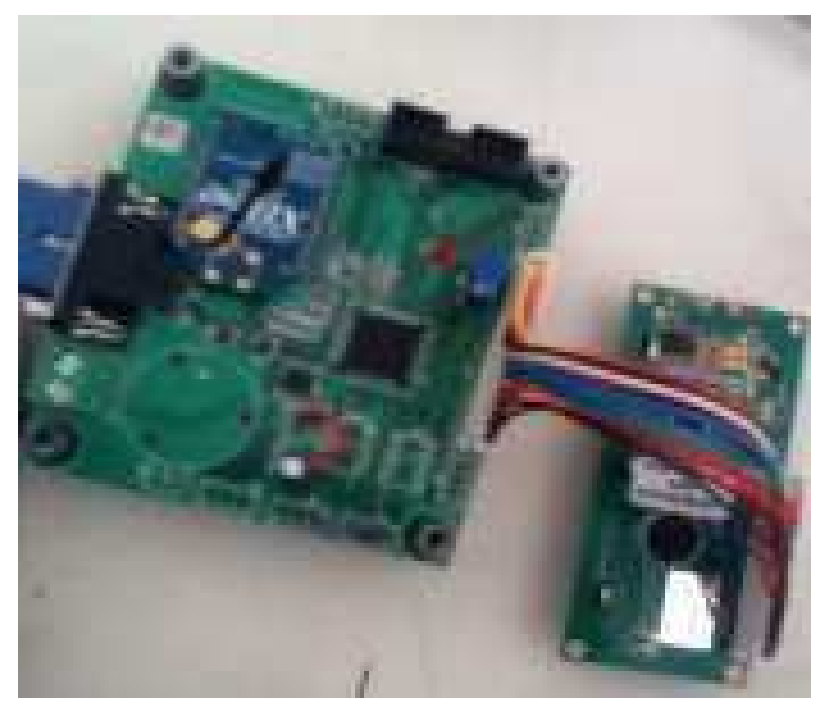

Figure 2: Video Sensor Node

Before doing the image processing steps, the smoke sensor node output is checked for the fire risk level. After image processing work done, it is compared and final decision is taken if it exceeds the threshold level. The figure 3 shows steps in the algorithms. The fire risk percentage is compared with the output image for the fire alarm activation. The false rate is zero when compared with smoke sensing process alone.

Table 1: Evaluation Results of Smoke Sensor System using Fuzzy Logic

\begin{tabular}{|c|c|c|c|c|c|}
\hline S.no & Temperature $\left({ }^{\circ} \mathbf{c}\right)$ & Smoke (ppm) & Light (lux) & Humidity (ppm) & Threat of Fire (\%) \\
\hline 1 & 20 & 30 & 300 & 80 & 27.8 \\
\hline 2 & 80 & 30 & 300 & 80 & 41 \\
\hline 3 & 20 & 80 & 300 & 80 & 33.8 \\
\hline 4 & 20 & 30 & 900 & 80 & 39.7 \\
\hline 5 & 20 & 30 & 300 & 80 & 30.3 \\
\hline 6 & 80 & 80 & 300 & 80 & 50 \\
\hline
\end{tabular}




\begin{tabular}{|c|c|c|c|c|c|}
\hline \multicolumn{7}{|c|}{ Table 1: Contd., } \\
\hline 7 & 80 & 80 & 800 & 80 & 53.6 \\
\hline 8 & 80 & 80 & 800 & 40 & 63.1 \\
\hline 9 & 100 & 80 & 800 & 40 & 70.1 \\
\hline
\end{tabular}

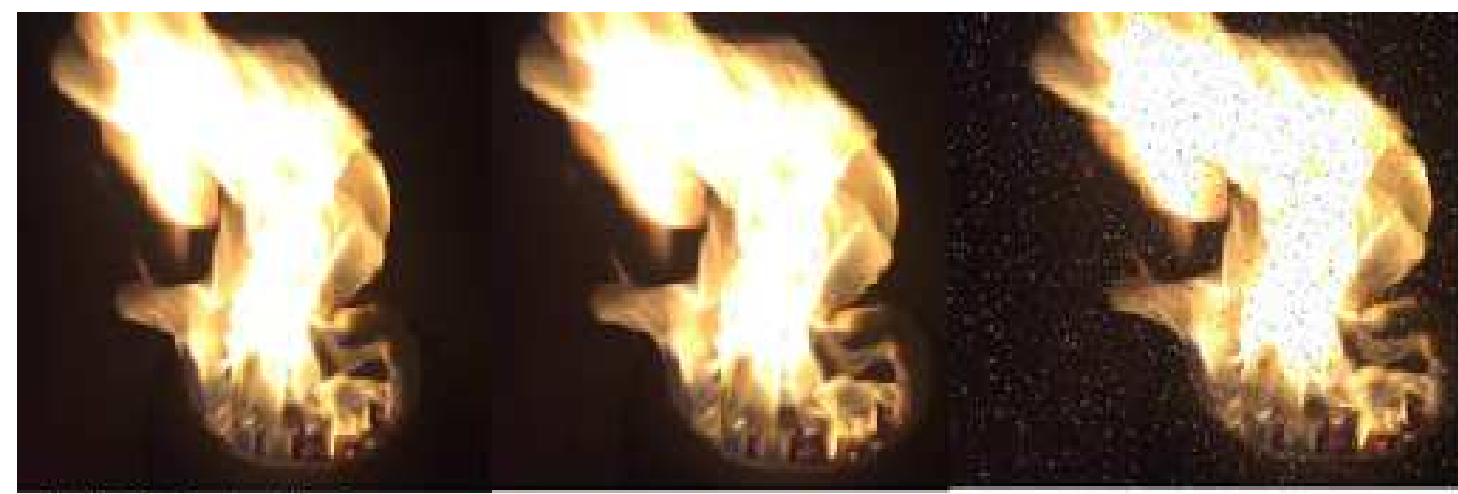

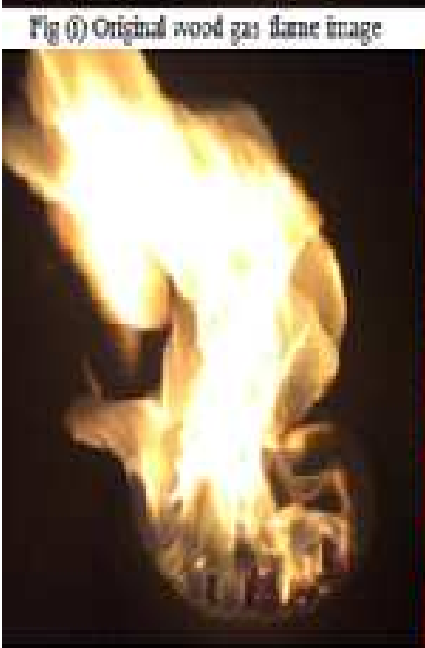

g (Ni) Smanti, thatp noile temoved irage

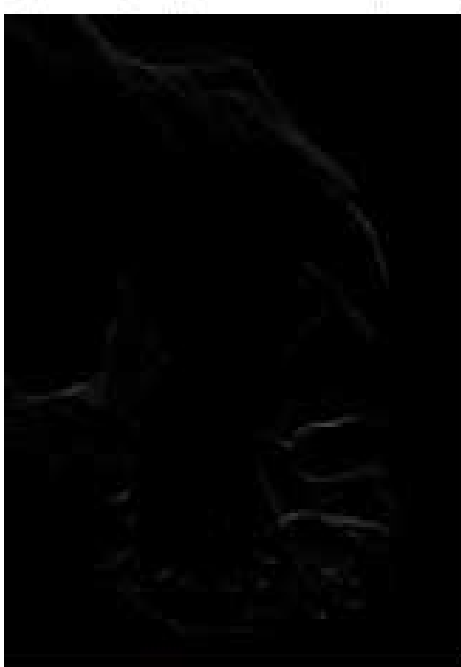

Fig.(vi) Vetical edpe imase
Fir (t) Recosd thane mage

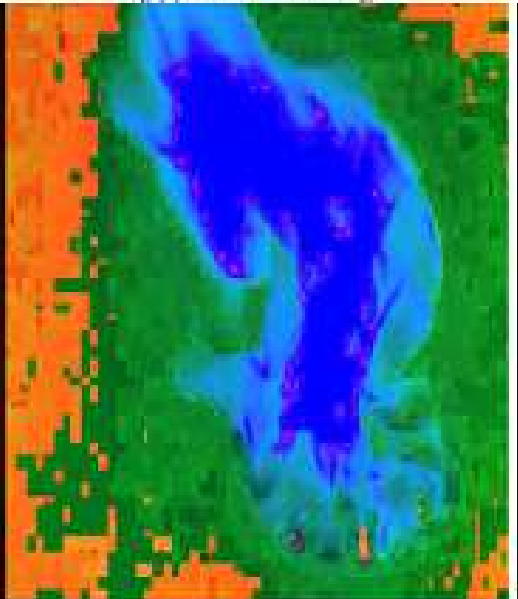

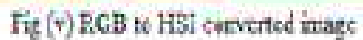

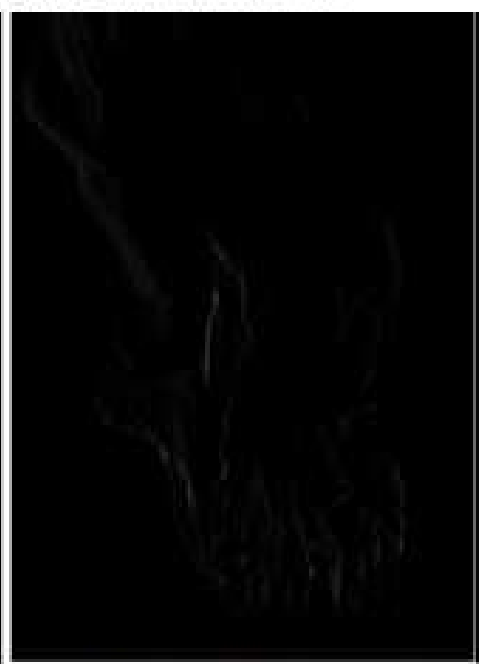

Fis (ive) Herrontal edt: mase
Fig.iil Sal and pegoes noise adted inaze

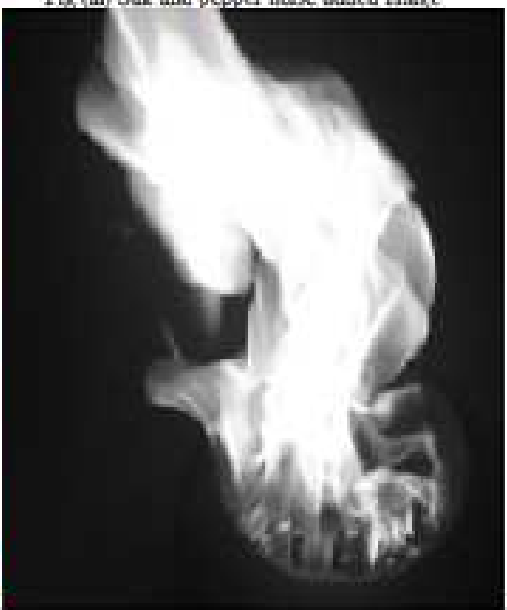

Fig (19i turinince, gay level mage

Figure (i):

Figure 3: Proposed Algorithm Technique Step Images and Final Output Image 


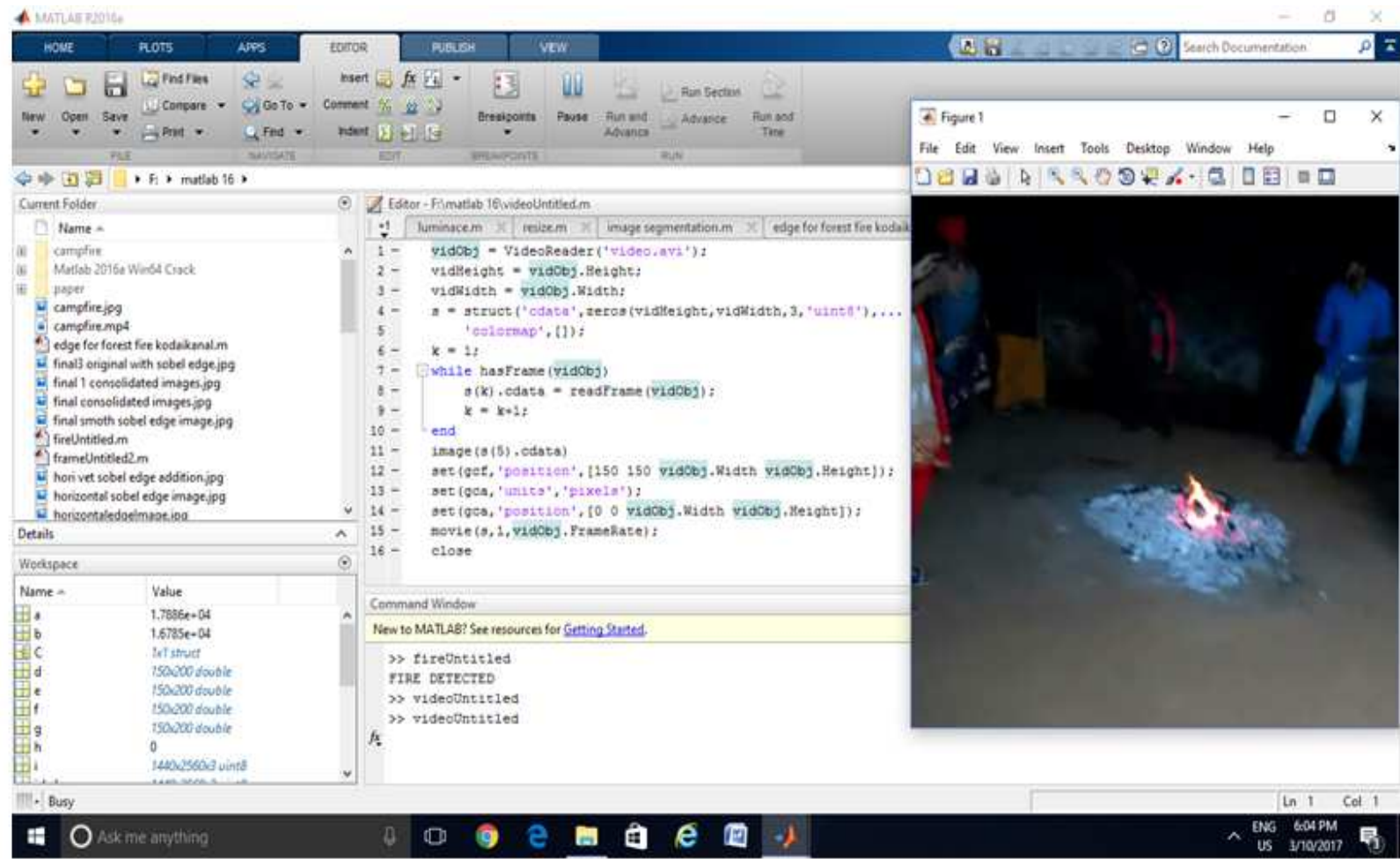

Figure 4: Converting Video into Frames

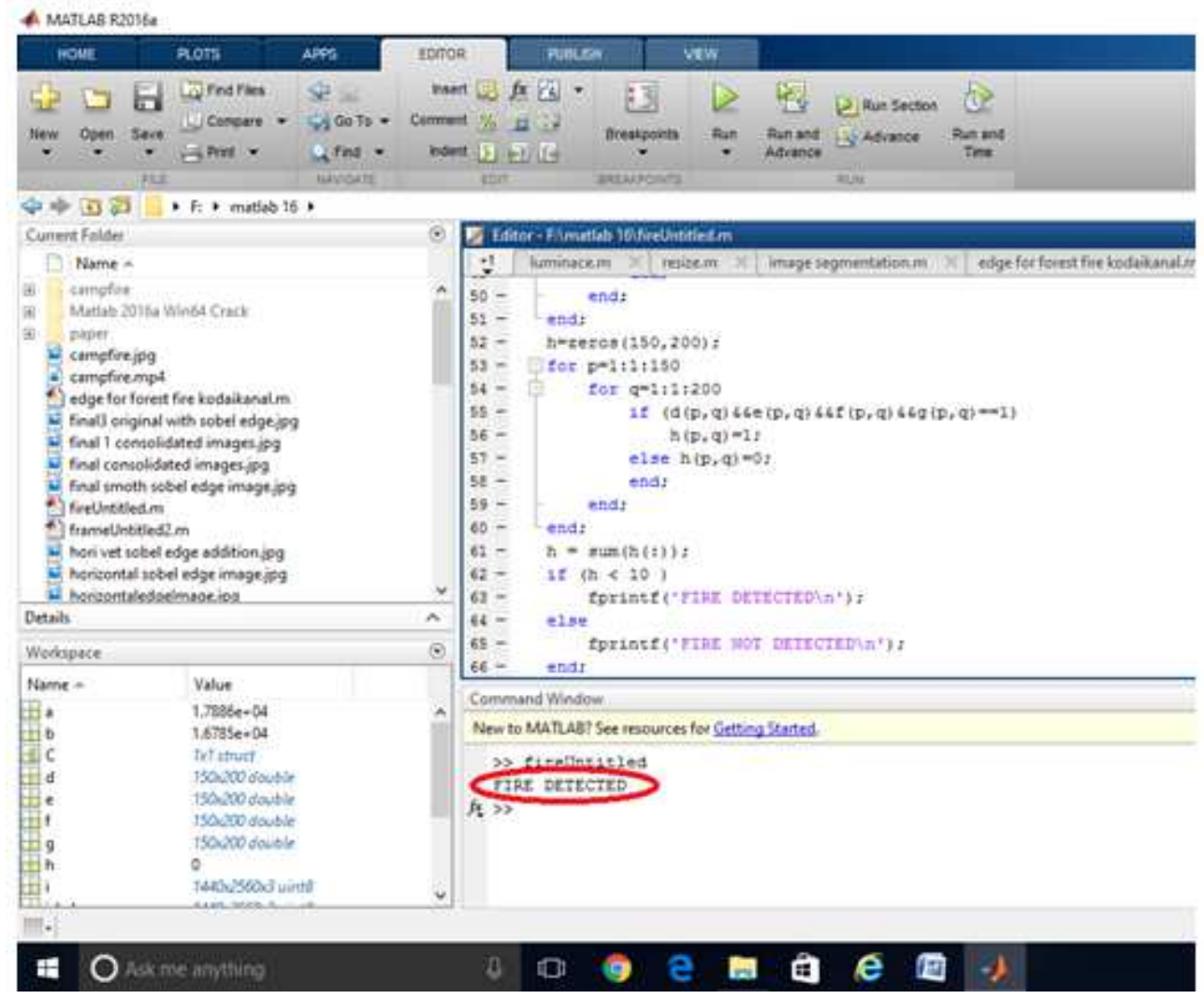

Figure 5: Fire Detected From the Video 
Fire pixels from the proposed technique were compared with human verified fire pixels. The accuracy is good for 90 fire images for 100 fire images. It also gives good accuracy up to 95 for 100 non fire images. For fire videos it gives notification within nano seconds. It means that the proposed system detects all fire video sequences and alert before disaster happens. In this first step is converting video into frame images which is shown in figure 4. The HSV/RGB/CMY color models are applied to detect fire location. The fire location has white pixels and it is compared with consecutive frame images to detect fire. If the fire detected from the video, it alerts sound and gives warning information to the user. The figure 5 gives information about fire detected from the video.

\section{CONCLUSIONS}

Based on the characteristics of fire and flame color, the system applies an image segmentation method to extract the suspected fire region. The color distribution algorithm, fire detection algorithm of cusp and dynamics algorithm and fuzzy logic to detect fire risk rate are executed parallel to detect the region. So, it greatly enhances the efficiency and the recognition rate of the algorithm to guarantee the real-time property of the fire monitoring system. Experimental results have demonstrated that the proposed algorithm is effective in identifying the edges of irregular flames for image compression. The advantage of this method is that the flame and fire edges detected are clear and continuous before sending the fire image information to monitoring station. This algorithm provides an useful addition to fire image processing and fire analysis in fire safety engineering. Better recognition results have been obtained through a large number of experiments. Zero false rate is obtained, when this technique is combined the output with the sensors such as temperature, humidity and smoke. The system uses HSV/RGB/CMY color models to separate orange, yellow, and high brightness from the background. Fire growth and spread is checked based on frame differences. The proposed system works very well when fire occurs, by providing significantly faster detection based on light detection and analysis. The obvious outcome is a significant reduction in loss of life and property. The overall accuracy of the experiments is around $90 \%$, demonstrating its effectiveness and usefulness. In future work, video fire smoke will be analyzed and the output is combined with video fire light to increase the accuracy till 100.

\section{ACKNOWLEDGMENT}

This work is supported by the University Grants Commission, Government of India under grant no. F.151/201415/PDFWM-2014-15-OB-TAM-24657

\section{REFERENCES}

1. S.R.Vijayalakshmi and S.Muruganand, "Real Time Monitoring of Wireless Fire Detection Node", Elsevier Procedia Technology 24 (2016) pp. $1113-1119$.

2. M. Morin at.el, "Computer-supported visualization of rescue operations", Safety Science, Vol.35, 2000, pp. 3-27.

3. Xue-gui Wan at.el , "A Novel Conceptual Fire Hazard Ranking Distribution System based on Multisensory Technology", Science Direct, Procedia Engineering, Vol.71, 2014, pp.567 - 576.

4. Konstantinous at.el, "An Automated Fire Detection and Alerting Application based on Satellite and Wireless Communications", 2010 5th Advanced Satellite Multimedia Systems Conference and the 11th Signal processing for space communications workshop.

5. Fatih Erden at.el, "Wavelet based flickering flame detector using differential PIR sensors", Fire Safety Journal Vol.53, 2012, pp.13-18. 
6. K.Jayaram, G.Ramachandran, L.Vasanth, and R.Nagarajan, "Adaptive edge detection algorithm for flame and fire images ", International journal of advance research in science and engineering, Vol. 3, No.01, pp. 84-90, January 2014.

7. Shaik Rasool and P.Pandarinath, " An Autoadaptive edge detection algorithm for flame and fire image processing", International journal of computer \& organization trends, Vol.3, No.11, pp.537-542, Dec 2013.

\section{AUTHORS DETAILS}

S.R.Vijayalakshmi * is a Post Doctoral research Fellow in Department of Electronics and Instrumentation in Bharathiar University. She received her B.Sc. M.Sc. M.Phil. and Ph.D in Electronics from the Bharathiar University and also received M.Sc. and M.Phil. in Computer Science from Avinashilingam University. She has 20 years of experience in the teaching and also in research. Her research interests include digital image processing, embedded systems, and wireless sensor networks. She completed one DST-WOS-A project funded by Government of India. (* corresponding author). She is an author of two books.

S.Muruganand is an Assistant Professor in Department of Electronics and Instrumentation in Bharathiar University. He received his M.Sc. Physics from Madras University, M.Ed. and Ph.D from Bharathiar University. He has 28 years of experience in the teaching and research field. His research interests include digital image processing, nano science, wireless sensor networks, thin films, and Microprocessors. He completed one UGC- Minor project funded by Government of India. He is author of many papers in the referred journals. 